\title{
Pharyngeal Neoplasm
}

National Cancer Institute

\section{Source}

National Cancer Institute. Pharyngeal Neoplasm. NCI Thesaurus. Code C3325.

A benign or malignant neoplasm that affects the nasopharynx, oropharynx, or hypopharynx. 